\title{
Dampening the fire to prevent surgery- and chemotherapy-induced metastasis
}

\author{
Esra Güç and Jeffrey W. Pollard \\ MRC Centre for Reproductive Health, Queen's Medical Research Institute, The University of Edinburgh, Scotland, United Kingdom.
}

\begin{abstract}
In this issue of the $J C I$, Panigrahy et al. demonstrate that preoperative administration of the antiinflammatory drug ketorolac or specialized proresolving mediators (SPM) called resolvins increases disease-free survival rates and prevents metastasis after surgery and chemotherapy in mouse models of cancer. The antitumor response was partially mediated by tumorspecific $\mathrm{T}$ cell immunity and immunological memory.
\end{abstract}

\section{When treatment also promotes} disease

A century after Virchow hypothesized the association between inflammation and cancer, it is becoming evident that inflammatory mediators lead to cancer progression and metastasis (1). Surgical incision- and chemotherapy-induced immune response has been known to enhance tumor-antigen recognition by $\mathrm{T}$ cells, yet paradoxically, the tumor killing mediates the release of acute inflammatory cytokines and prostaglandins, which helps tumor dissemination and metastasis $(2,3)$. Moreover, although neoadjuvant and adjuvant chemotherapy are the most potent methods for cancer treatment, both clinical and experimental studies suggest that chemotherapy can induce the incidence of premetastatic niche formation by increasing the release of inflammatory factors and extracellular vesicles from the primary tumor as well as awakening dormant metastasized cells (4-6).

Prostaglandins and leukotrienes are actively produced lipids in chronic inflammation and cancer. Cyclooxygenase-2-mediated (COX-2-mediated) prostaglandin $\mathrm{E}_{2}\left(\mathrm{PGE}_{2}\right)$ production is increased during tumorigenesis, and its secretion is induced by several immune cells, including macrophages and neutrophils (7). Epidemiologic and clinical metaanalysis provides strong evidence that the use of COX-2-selective or COX-1/ COX-2-inhibiting NSAIDs increases disease-free survival rate and reduces cancer recurrence $(8,9)$. Nevertheless, this association is not established for all types of cancer, and especially, the chemopreventive role of NSAIDs remains controversial because of the toxicity caused by long-term inhibition of COX and prostaglandins $(10,11)$. The antitumoral role of COX-1-inhibiting NSAIDs is less well studied, since this pathway is not overexpressed during tumor progression and it plays an important role in homeostasis (12).

\section{Preoperative inflammation inhibition increases metastasis-free survival}

In this issue of the JCI, a new study by Panigrahy and colleagues brings a different perspective on the role of the COX-1 pathway in cancer therapy (13). The study addresses the paradoxical finding that surgery and chemotherapy, while often effective therapeutically, can also lead to an increase in metastasis, a finding that has been attributed to inflammatory responses. The authors first demonstrate how surgical trauma and chemotherapy applied to tumors that are at or below the threshold of growth enhanced tumor escape

Related Article: p. 2964

Conflict of interest: The authors have declared that no conflict of interest exists.

as well as metastasis. Inhibition of inflammation by the administration of a potent NSAID ketorolac, when given preoperatively but not postoperatively, increased metastasis-free survival. Similar results were obtained with their use in chemotherapy.

To better understand whether this effect was selective to COX-1 inhibition, the authors tested various COX-1, COX-2, and COX-1/2 inhibitors including some of the commonly used NSAIDs, such as indomethacin, aspirin, and ibuprofen. These studies indicated that COX-1 inhibitors, but not COX-2 selective inhibitors, induced an antitumor effect when applied at the preoperative stage. Interestingly, the authors demonstrated that the effects of COX-1 inhibition were diminished when the COX-2 pathway and prostaglandin $\left(\mathrm{PGE}_{2}\right.$ ) production were blocked in the Lewis lung carcinoma (LLC) model. Thromboxane $\mathrm{A}_{2}\left(\mathrm{TXA}_{2}\right)$ downstream from COX- 1 is a potent vasoconstrictor and induces platelet aggregation, a process that is known to help metastatic tumor seeding and cancer cell survival in mouse models. Preoperative ketorolac decreased $\mathrm{TXA}_{2}$ production, and administration of a TXA 2 inhibitor also showed antitumor effects similar to those of ketorolac, suggesting that targeting this pathway as precancer treatment might be important in preventing metastatic seeding (Figure 1).

Although Serhan and his group described the role of resolvins and lipoxins in the resolution of inflammation in 2002 (14), little is known about their functions in cancer inflammation. Yet administering resolvins as a cancer treatment provides significant improvement. A recent study by Panigrahy et al. explored this mechanism and showed that resolvins enhance the debris-cleaning function of macrophages and prevent cell-mediated inflammatory response and tumor progression (15). Similarly, in this current paper, preoperative resolvin administration drastically prolonged tumor-free survival after tumor resection. However, whether this result is correlated with resolvin-mediated 

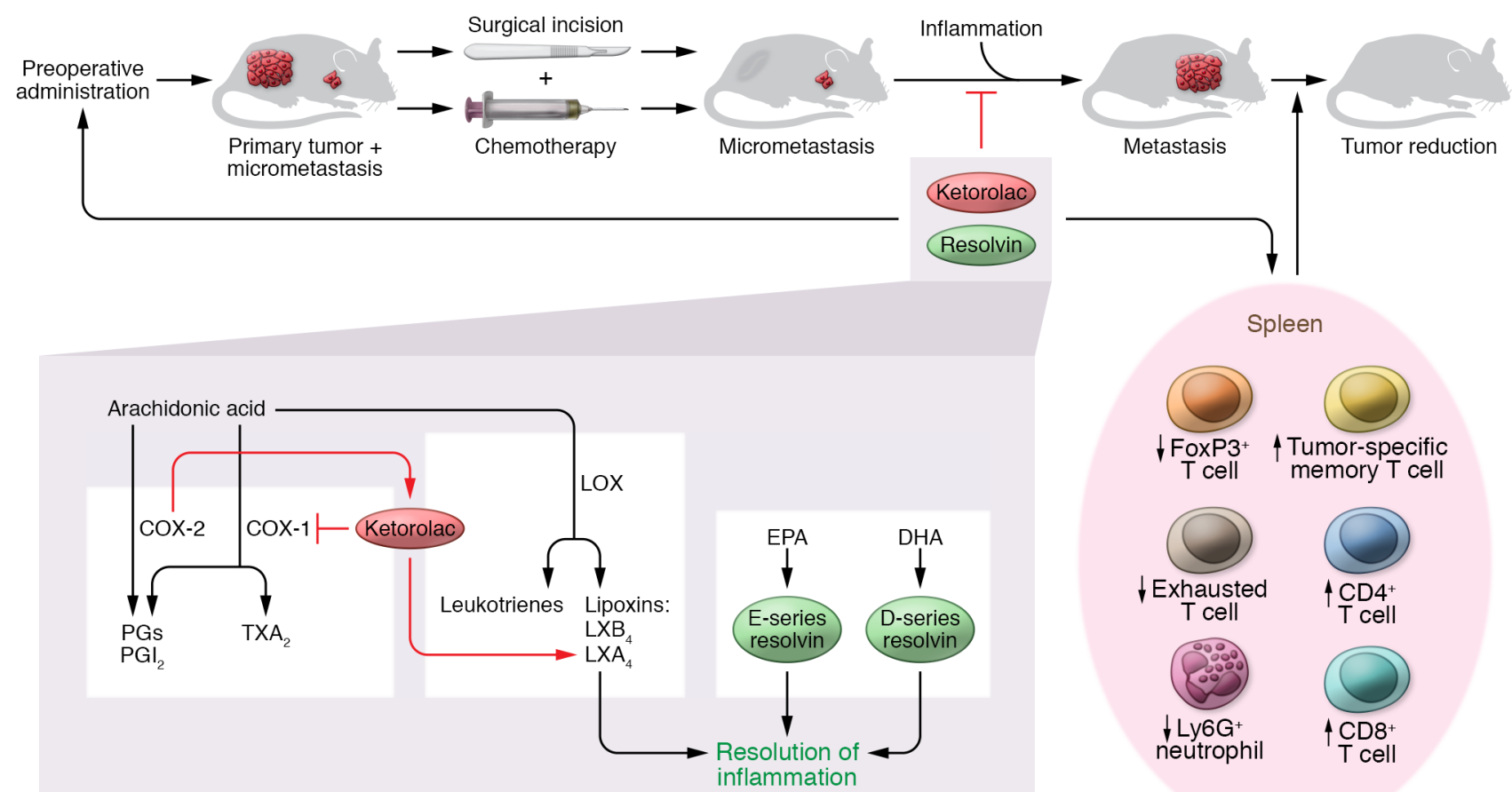

Figure 1. Surgical excision and chemotherapy induce inflammation-mediated metastasis in mouse models of cancer that can be blocked with ketorolac and resolvins. Preoperative administration of the antiinflammatory NSAID ketorolac and the SPM called resolvin increases disease-free survival rates and prevents metastasis in mouse tumor models. Ketorolac inhibits the COX-1 pathway and formation of platelet aggregation by blocking TXA ${ }_{2}$ production. COX-2/PGE 2 activity is required when ketorolac is administered preoperatively. Ketorolac also increases the production of an SPM called lipoxin A (LXA S $_{4}$ that plays a role in the antitumor function of ketorolac. Resolvins are produced from omega-3 fatty acid metabolism; however, in this study, resolvins were exogenously administered at the preoperative stage and increased cancer-free survival in mouse models. Preoperative ketorolac administration increased $\mathrm{CD}^{+}$and $\mathrm{CD}^{+} \mathrm{T}$ cells and immunological memory, decreased Foxp $3^{+}$regulatory $\mathrm{T}$ cells, and exhausted $\mathrm{T}$ cells in the spleen. These immunological changes mediate the antitumor action of this inhibitor. PGs, prostaglandin E2, D2 and F2 alpha; PGI, prostacyclins; LOX, lipoxygenase; EPA, eicosapentaenoic acid; DHA, docosahexaenoic acid.

debris cleaning or through the resolution of inflammation and how this affects the prometastatic infiltration of inflammatory monocytes in metastatic organs are open questions that should be investigated in future studies.

The authors investigated how ketorolac and resolvins might act by examining the role of splenic antitumor immune responses. Preoperative administration of ketorolac increased splenic $\mathrm{CD}^{+}$and $\mathrm{CD}^{+} \mathrm{T}$ cells, whereas Foxp-3, a marker of T regulatory cells, was decreased. Previous studies targeting the COX-2 pathway and $\mathrm{PGE}_{2}$ production also demonstrated a reduction of $\mathrm{CD} 4^{+} \mathrm{Foxp}-3^{+} \mathrm{T}$ cells $(16,17)$, suggesting that both COX-1 and COX-2 inhibition targets immunosuppressive tumor mechanisms. The authors also showed that the antitumor effect of ketorolac is $\mathrm{T}$ cell dependent. Importantly, ketorolac acts synergistically with the immune checkpoint inhibitor, anti-PD1 antibody, with survival rates drastically increasing in mice administered such combination therapy.

Furthermore, ketorolac also mediated immunological memory. Months after tumor resection, ketorolac-treated mice rechallenged with the same tumor cell type rejected those cells; however, this effect was tumor specific, indicating the acquisition of immunity. Adoptive $\mathrm{CD} 8^{+} \mathrm{T}$ cell transfer from this group of tumor-challenged mice into naive mice also gave them antitumor immunity to the comparable tumor type. This finding is striking and highlights that short-term NSAID administration just before surgical incision affects the longterm antitumor immune responses.

To investigate this phenomenon further, the authors performed single-cell transcriptomic analysis of splenic immune cells seven days after the surgical incision. While the depth of sequence and analysis was relatively slight, the results confirmed their previous histological analysis and showed that ketorolac increased the number of $\mathrm{T}$ cells in the spleen after tumor resection. Gene ontology of $\mathrm{T}$ cell clusters suggested that ketorolac helps to rescue $\mathrm{T}$ cell exhaustion and increase the number of memory $T$ cells. Although a drastic change in the B cell population was detected after ketorolac treatment, the role of B cells in developing immunological memory was not explored. This group also performed a more comprehensive analysis of $\mathrm{Ly} 6 \mathrm{G}^{+}$ granulocytes and showed that this population is decreased in ketorolac-treated mice concurrent with decreased MMP-9, $\mathrm{NF}-\kappa \mathrm{B}$, and PI3K activation. These genes are associated with protumoral granulocytes that suppress $\mathrm{T}$ cell proliferation and antitumor immunity. Together, these data strongly suggest that granulocytic immunosuppression was removed by ketorolac.

This study is valuable in terms of understanding the impact of NSAIDs on cancer treatment. Importantly, it emphasizes the need to understand biological responses to inflammation that can be protumoral in 
order to effectively suppress it. In addition, this study emphasizes the need for the correct timing required to inhibit such inflammatory responses to have a therapeutic impact. It also suggests that standard-ofcare treatments with the appropriate antiinflammatory reagents might unleash a $\mathrm{T}$ cell response that ablates dormant metastatic cells and micrometastases. If this can be translated from mouse models into the clinic, then it could revolutionize treatments. Notably, the COX-2 pathway ameliorated the effect of ketorolac, indicating the need for correct combinations of COX-1/COX-2 inhibitors even within this NSAID class.

\section{Conclusions and remaining questions}

While an important first step, this study leaves many unanswered questions that will need to be investigated before moving from mouse models to clinical trials. Foremost among these is the sole use of xenografted cancer models that are highly immunogenic and injected subcutaneously. While this system is a proof of principle, these are not true autochthonous cancers that are heterogeneously evolving structures with complex and continuously changing microenvironments. In addition, it needs to be established how resolvins and ketorolac launch the antitumoral immune response and how these molecules rescue exhausted $\mathrm{T}$ cells and synergize with anti-checkpoint inhibitors. At present, the changes in immune cells in this study were analyzed only at low resolution from the spleen, but not lymph nodes or target tissues, so studies in greater depth are in order. It will also be fascinating to understand the mechanisms of the response in light of other recent observations in this area. For example, studies have shown that chemotherapeutic stress on cancer cells induces the production of microvesicles that prime premetastatic sites (5). Are the beneficial effects of resolvins and ketorolac mediated by the resolution of inflammation and subsequent blocking of microvesicle formation and/or cancer cell homing or are they solely mediated through effects on immune cells that kill metastatic tumor cells?

\section{Acknowledgments}

This work was supported by the Wellcome Trust (101067/Z/13/Z), MRC Centre grant MR/NO22556/1, and Cancer Research UK (CRUK) grant C17950/A26783 (to JWP).

Address correspondence to: Jeffrey W. Pollard, MRC Centre for Reproductive Health, Queen's Medical Research Institute, Edinburgh BioQuarter, 47 Little France Crescent, EH16 4TJ, Edinburgh, Scotland, United Kingdom. Phone: 44.0.1312426231; Email: Jeff.Pollard@ed.ac.uk.

1. Balkwill F, Mantovani A. Inflammation and cancer: back to Virchow? Lancet. 2001;357(9255):539-545.

2. Tohme S, Simmons RL, Tsung A. Surgery for cancer: a trigger for metastases. Cancer Res. 2017;77(7):1548-1552.

3. Krall JA, et al. The systemic response to surgery triggers the outgrowth of distant immune-controlled tumors in mouse models of dormancy. Sci Transl Med. 2018;10(436):eaan3464.

4. Middleton JD, Stover DG, Hai T. Chemotherapy-exacerbated breast cancer metastasis: a paradox explainable by dysregulated adaptiveresponse. Int JMol Sci. 2018;19(11):E3333.
5. Keklikoglou I, et al. Chemotherapy elicits pro-metastatic extracellular vesicles in breast cancer models. Nat Cell Biol. 2019;21(2):190-202.

6. Vyas D, Laput G, Vyas AK. Chemotherapyenhanced inflammation may lead to the failure of therapy and metastasis. Onco Targets Ther. 2014;7:1015-1023.

7. Kalinski P. Regulation of immune responses by prostaglandin E2. J Immunol. 2012;188(1):21-28.

8. Gurpinar E, Grizzle WE, Piazza GA. NSAID inhibit tumorigenesis, but how? Clin Cancer Res. 2014;20(5):1104-1113.

9. Zhao X, Xu Z, Li H. NSAIDs use and reduced metastasis in cancer patients: results from a meta-analysis. Sci Rep. 2017;7(1):1875.

10. Setiawan VW, et al. Use of nonsteroidal antiinflammatory drugs and risk of ovarian and endometrial cancer: the Multiethnic Cohort. Cancer Epidemiol Biomarkers Prev. 2012;21(9):1441-1449.

11. Gurpinar E, Grizzle WE, Piazza GA. COX-independent mechanisms of cancer chemoprevention by anti-inflammatory drugs. Front Oncol. 2013;3:181.

12. Wang D, Dubois RN. Eicosanoids and cancer. Nat Rev Cancer. 2010;10(3):181-193.

13. Panigrahy D, et al. Preoperative stimulation of resolution and inflammation blockade eradicates micrometastases. JClin Invest. 2019;129(7):2964-2979.

14. Serhan CN, et al. Resolvins: a family of bioactive products of omega-3 fatty acid transformation circuits initiated by aspirin treatment that counter proinflammation signals. J Exp Med. 2002;196(8):1025-1037.

15. Sulciner ML, et al. Resolvins suppress tumor growth and enhance cancer therapy. JExp Med. 2018;215(1):115-140.

16. Sharma S, et al. Tumor cyclooxygenase-2/ prostaglandin E2-dependent promotion of FOXP3 expression and $\mathrm{CD} 4{ }^{+} \mathrm{CD} 25^{+} \mathrm{T}$ regulatory cell activities in lung cancer. Cancer Res. 2005;65(12):5211-5220.

17. Hibino S, et al. Inhibition of $\mathrm{Nr} 4$ a receptors enhances antitumor immunity by breaking Treg-mediated immune tolerance. Cancer Res. 2018;78(11):3027-3040. 